Related content

\section{Pedagogical models of surface mechanical wave propagation in various materials}

To cite this article: L Bellomonte et al 2004 Eur. J. Phys. 25745

View the article online for updates and enhancements.

\begin{abstract}
How did Roman buildings survive earthquakes?

C Fazio, R M Sperandeo-Mineo and G Tarantino

Measuring longitudinal wave speed in solids

C Fazio, I Guastella, R M SperandeoMineo et al.

- Strong ground motion simulation for seismic hazard assessment in an urban area

Zhixin Zhao, Zhao Zhao, Jiren Xu et al.
\end{abstract}

\section{Recent citations}

Measuring longitudinal wave speed in solids: two methods and a half C Fazio et al

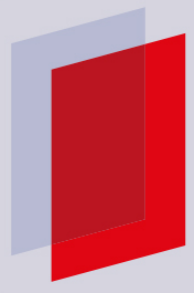

\section{IOP ebooks}

Bringing you innovative digital publishing with leading voices to create your essential collection of books in STEM research. Start exploring the collection - download the first chapter of every title for free. 


\title{
Pedagogical models of surface mechanical wave propagation in various materials
}

\author{
L Bellomonte, C Fazio, R M Sperandeo-Mineo \\ and G Tarantino
}

GRIAF (Research Group on Teaching/Learning Physics), Dipartimento di Fisica e Tecnologie Relative, Università di Palermo, Italy

Received 5 March 2004

Published 25 August 2004

Online at stacks.iop.org/EJP/25/745

doi:10.1088/0143-0807/25/6/006

\begin{abstract}
We report on a teaching approach oriented to the understanding of some relevant concepts of wave propagation in solids. It is based on simple experiments involving the propagation of shock mechanical waves in solid slabs of various materials. Methods similar to the generation and propagation of seismic waves are adopted. Educational seismometers, interfaced with computers, are used to detect and visualize the shock waves and to analyse their propagation properties. A qualitative discussion of the results concerning the propagation and the attenuation of the waves allows us to draw basic conclusions about the response of the matter to solicitation impacts and their propagation.
\end{abstract}

(Some figures in this article are in colour only in the electronic version)

\section{Introduction}

In the past few years, many papers and conferences have addressed the need for motivating students in physics-learning through the introduction of teaching approaches involving relevant real-life problems. Various approaches have been developed; they involve environmental physics [1] or medical physics [2] as well as integrated curricula [3].

In this paper, we describe an activity-based approach aimed at stimulating a meaningful understanding of mechanical wave propagation in the earth science context. The main feature of the present approach is the analysis of some characteristics of the mechanical waves by taking as a starting point a phenomenon familiar to pupils: the earthquake. Direct evidence of earthquakes comes from the damage produced by the phenomenon and seismographic registrations frequently reported on TV networks and in newspapers. In order to focus on the characteristics of energy propagation, it must be made clear to students that there exist different kinds of seismic waves: P-waves, $\mathrm{S}$-waves and surface waves; these waves are described (with 
useful visuals) in many Web sites (see, for example [4]). The P-waves are longitudinal waves, whereas the $\mathrm{S}$-waves are transverse waves which travel only in solids and move more slowly than the P-waves but are more powerful and often much more destructive. When the energy of an earthquake reaches the surface of the earth, it produces surface waves. These waves (called Love and Rayleigh waves [4]) are often the strongest waves recorded by the seismograms. They travel a little slower than S-waves so they tend to reach the seismograph just after the $\mathrm{S}$-waves. Surface waves churn over and under like rolling ocean waves, causing elliptic motion of the earth surface layers (they are rolling waves that make one feel as if one is struggling to keep one's balance on a ship in the ocean). Though these waves move more slowly than P- or $\mathrm{S}$-waves, they are much more destructive and are often those responsible for building collapse.

Recent observations have shown that the seismic response of nearby buildings can be, under appropriate circumstances, quite different. As an example, the Marco Aurelio and the Trajan columns, in the city of Rome, are identical in terms of age and are located only $800 \mathrm{~m}$ away, but only the former has suffered serious earthquake damage. This seems to result from the fact that while the bedrock of the city is made up of rather homogeneous Pliocene clays extending hundreds of metres down, the two columns are found to rest on superficial terrains with very different mechanical characteristics. A new branch of seismology has been developed, having as an objective the analysis of spatial variation of earthquake ground motion (SVEGM) (see, for example, the manuscript corresponding to seminars presented by Professor R S Harichandran at University of New South Wales, Sidney, Australia [5]). It has been shown that differences in the local soil conditions and in the geological make-up of the subsoil may alter the amplitude and frequency content of the bedrock motion.

For these reasons, we have focused our attention on some experiments aimed to analyse the properties of weak mechanical shock waves in slabs of different materials, in order to demonstrate the existence of differences in the spectral content and energy absorption of surface waves in different media. Afterwards, some relevant concepts of mechanical wave propagation are introduced in order to understand the experimental results. We have used commercial Real Time Laboratory (RTL) ${ }^{1}$ systems (also known as Micro-Computer Based Laboratory (MBL)) that comprehend the sensors and computer interfaces carried by userfriendly software that is able to collect, graph and analyse data as well as to export them in the format used by usual spreadsheet software or other data-analysis software.

A simplified version of this approach, addressed to high school pupils, has been published elsewhere [8]; here we report a possible development for college students.

\section{Description of the experiments}

We have used educational seismometers ${ }^{2}$ based on the Faraday-Lenz law; in these systems, a coil, secured by four springs, oscillates when hit by an incoming disturbance. Since the coil is bound to move in a magnetic field, the induced voltage is proportional to its velocity. The seismometers are anchored to slabs of different materials and shock waves are produced by inducing a disturbance through a stroke produced by a steel sphere $(97 \mathrm{~g})$ falling from a fixed height $(1 \mathrm{~m})$. The electric signals induced in the oscillating coils are first amplified and

1 RTL systems are widely used nowadays in science education, at high school and college level, because they enable students to observe and work out data in real time, to easily analyse data and to be confronted with predictions. Several commercial RTL systems are available on the market; we have used systems produced by Vernier Software and Technology [6] and Pasco Scientific [7].

2 Several types of seismometers are available in the educational laboratory market. We have used the model MT 15479, produced by Pierron Enterprises: http://www.pierron.com. 
(a)
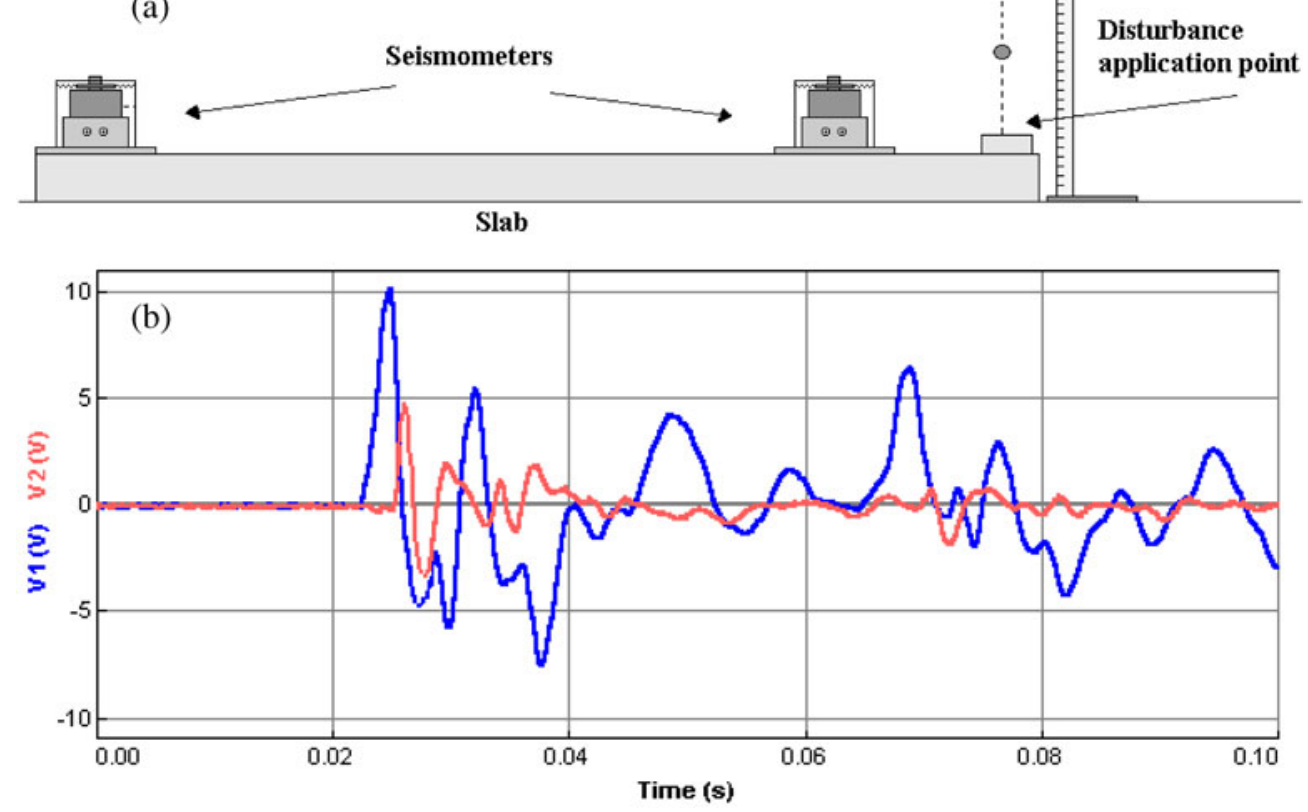

Figure 1. (a) Experimental apparatus. The disturbance is produced by the collision of an iron sphere falling from a given height. Slab dimensions were about $2.5 \times 0.4 \times 0.02 \mathrm{~m}^{3}$. (b) Typical signals registered by the two seismometers.

then sent to the inputs of the computer interface. Our experimental apparatus is a simplified version of other apparatus reported in the literature [9].

In order to analyse the characteristics of the propagation of a mechanical shock wave in different materials, two seismometers (with identical responses to equal disturbances) were anchored, at known distances, to slabs (of the same length) of steel, aluminium alloy, wood or marine sand ${ }^{3}$. Figure 1(a) shows the usual set-up of an experiment and figure 1(b) reports the typical signals registered by the two seismometers, located at the two extremes of the slab. Experimental values of the velocities of such shock waves, obtained using this experimental set-up, have been reported elsewhere [8]. We here analyse the time dependence of the amplitudes and shapes of the two signals and their related spectral compositions.

For each slab type, the experiments were repeated many times to ensure reproducibility under the same conditions. It turned out that the reproducibility was within 5\%. The signals registered by the two seismometers were different in both amplitude and shape, as expected. Changes in the falling height of the ball or in its mass determine variations in the amplitude of the signals, although they leave their spectral contents unchanged .

Working under identical experimental conditions (i.e. same ball, falling from the same height) the differences in amplitudes recorded by the first seismometer can be attributed to the typology of the coupling between each slab and the detector. The values of the amplitude registered by the first seismometer decrease for seismometers anchored to slabs of steel, aluminium, wood and sand, respectively.

Moreover, the signal attenuations at the two seismometers anchored to each slab are peculiar to each slab: the amplitude damping appears to depend on the material (it is higher

3 The sand was actually arranged in a long wooden container of geometrical dimensions similar to those of the slabs. 


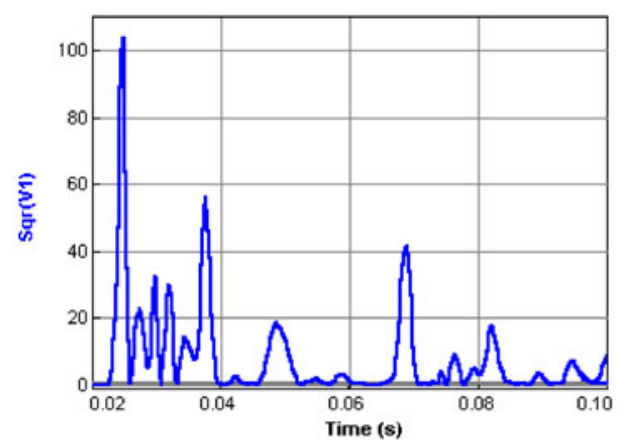

(a)
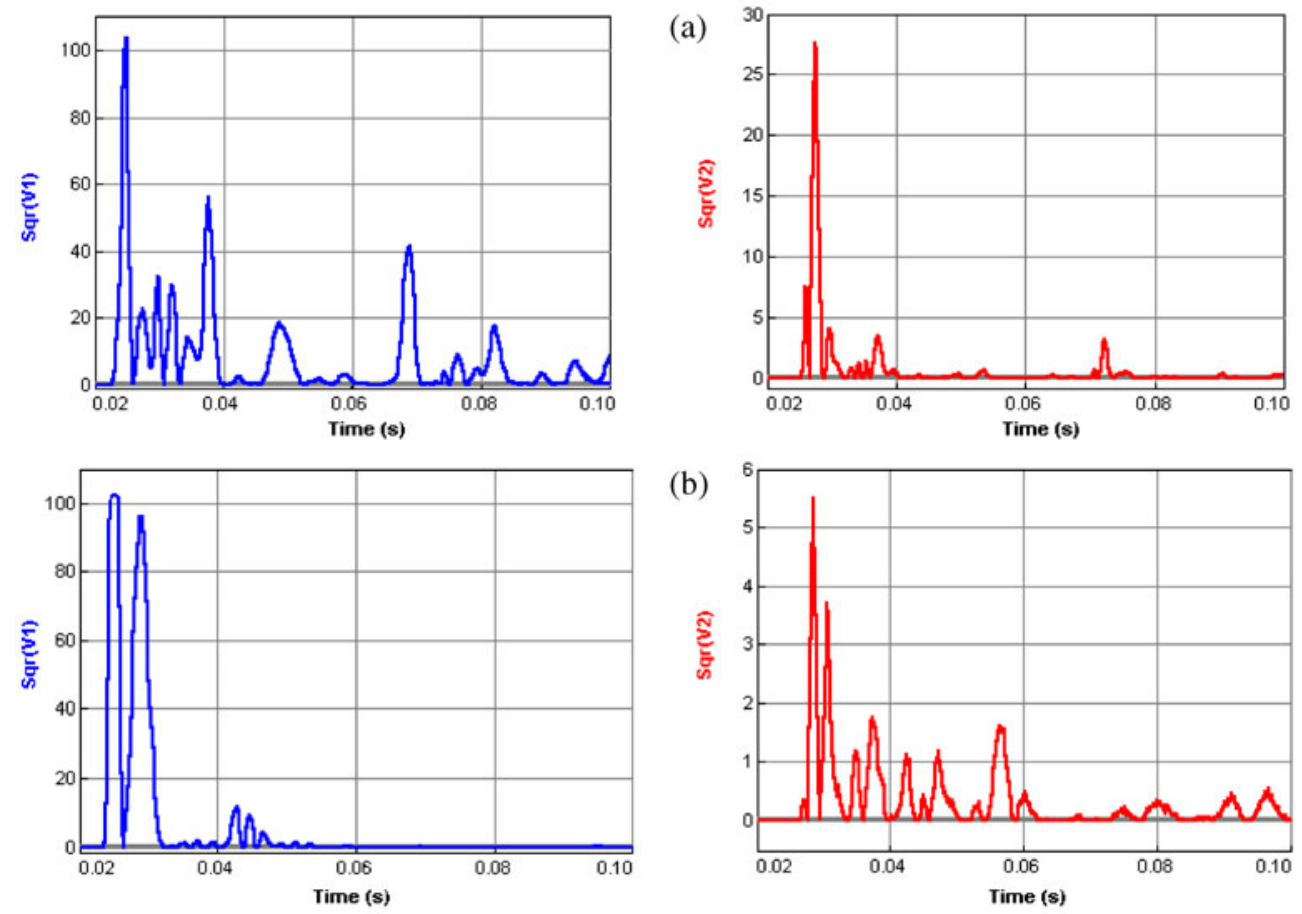

(b)
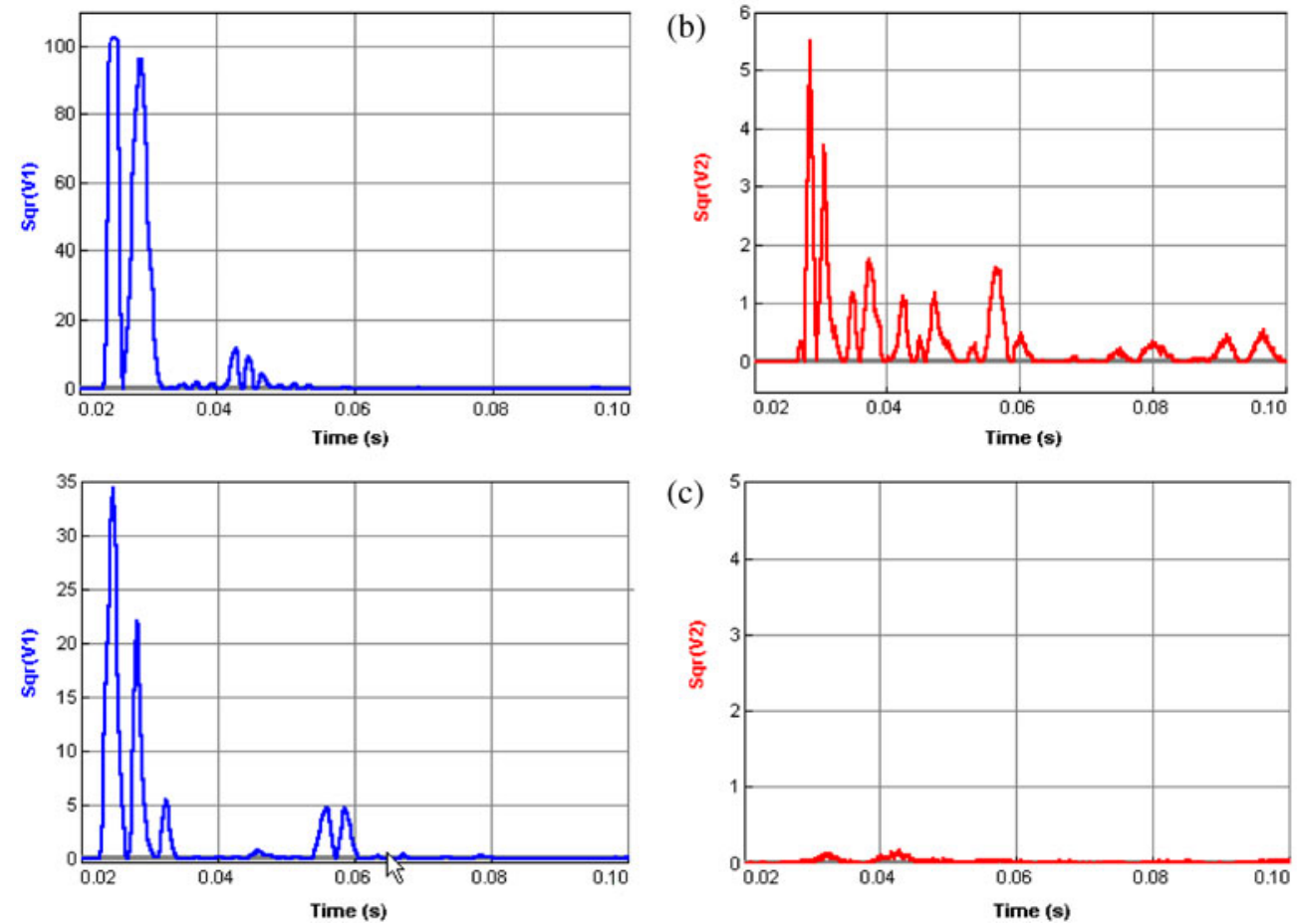

Figure 2. Time dependences of the amplitude squares of the two seismometer signals in aluminium alloy (a), wood (b) and sand (c).

in sand than in wood, aluminium or steel). This attenuation is also frequency dependent, so that the signal shape and the spectral distribution are sample dependent. In order to analyse the attenuation of signals we have studied their power content. To determine the effects of the material on the spectral distribution we have performed the Fourier analysis [10] (see the appendix).

The power content of a signal is proportional to the square amplitude of the signal itself; so, on taking into account the time dependence of the square amplitude of each seismometer signal (figure 2) and calculating the corresponding integrals over equal time intervals, we are able to evaluate the energy transmitted by the propagating wave to the first seismometer $E_{1}$ and to the second one $E_{2}$. The ratios $E_{1} / E_{2}$ allow us to evaluate the damping of different media (see table 1). 
Table 1. Mean damping values $\left\langle E_{1} / E_{2}\right\rangle$ of shock waves in sand, wood, aluminium alloy and steel. Mean values refer to 10 measurements and errors are taken as standard deviations of each set of measurements.

\begin{tabular}{lr}
\hline Medium & $\left\langle E_{1} / E_{2}\right\rangle$ \\
\hline Sand & $40 \pm 7$ \\
Wood & $15 \pm 3$ \\
Aluminium alloy & $9 \pm 2$ \\
Steel & $8 \pm 2$ \\
\hline
\end{tabular}

Further information about the shock wave propagation in different materials can be gained by analysing their spectral composition. The proper algorithm is the well-known Fourier transform [10] (see the appendix). An improvement consists in the fast Fourier transform (FFT) devised to appreciably reduce the amount of computational time [11]. This kind of data analysis is actually available in all commercial software for data logging. Various algorithms $[12,13]$ are available (see the appendix) and adaptable to commercial spreadsheets.

Comparison of the signal spectra detected by the two seismometers in different materials (figures 3(a)-(c)) indicates that the values of the harmonic components appreciably decrease with distance from the shock point and the spectral compositions modify too. Both aspects depend on the material. In particular,

- in an aluminium alloy slab, the reduction factor is 4 for lower frequencies (less than $200 \mathrm{~Hz}$ ) and 2 for higher frequencies, approximately; the same effect has been detected in steel slabs;

- in a wood slab, the reduction is almost frequency independent;

- in a sand substrate, heavy attenuation occurs: only frequencies lower than $70-100 \mathrm{~Hz}$ are transmitted, although with heavy attenuation.

These results indicate that the structure of the material plays an important role in mechanical wave propagation. Moreover, the behaviour in metals shows a selective damping of the frequencies.

The main experimental results can be summarized as follows:

- shock waves propagating in the different materials are subject to different energy attenuations;

- metals show a selective damping of the frequencies.

In order to give simple explanation of the behaviour of matter subjected to mechanical perturbations represented by a shock wave, a careful analysis of the materials used in the experiments is necessary. This analysis makes possible the introduction of relevant characteristics of waves propagation and related physics concepts.

\section{Result discussion}

The above experimental results may be considered as a useful tool in the process of introducing the students to the propagation of the mechanical waves and to qualitatively explain the dependence of their peculiarities, such as attenuation, spectral content etc, on the characteristics of the media in which they propagate. It has been shown $[15,16]$ that many students, even at college level, face difficulties in really understanding what is a wave and tend to associate the concept of propagation of matter with that of wave propagation. The simultaneous display of the shock pulses registered by the two seismometers, placed at given distances on the slabs, 

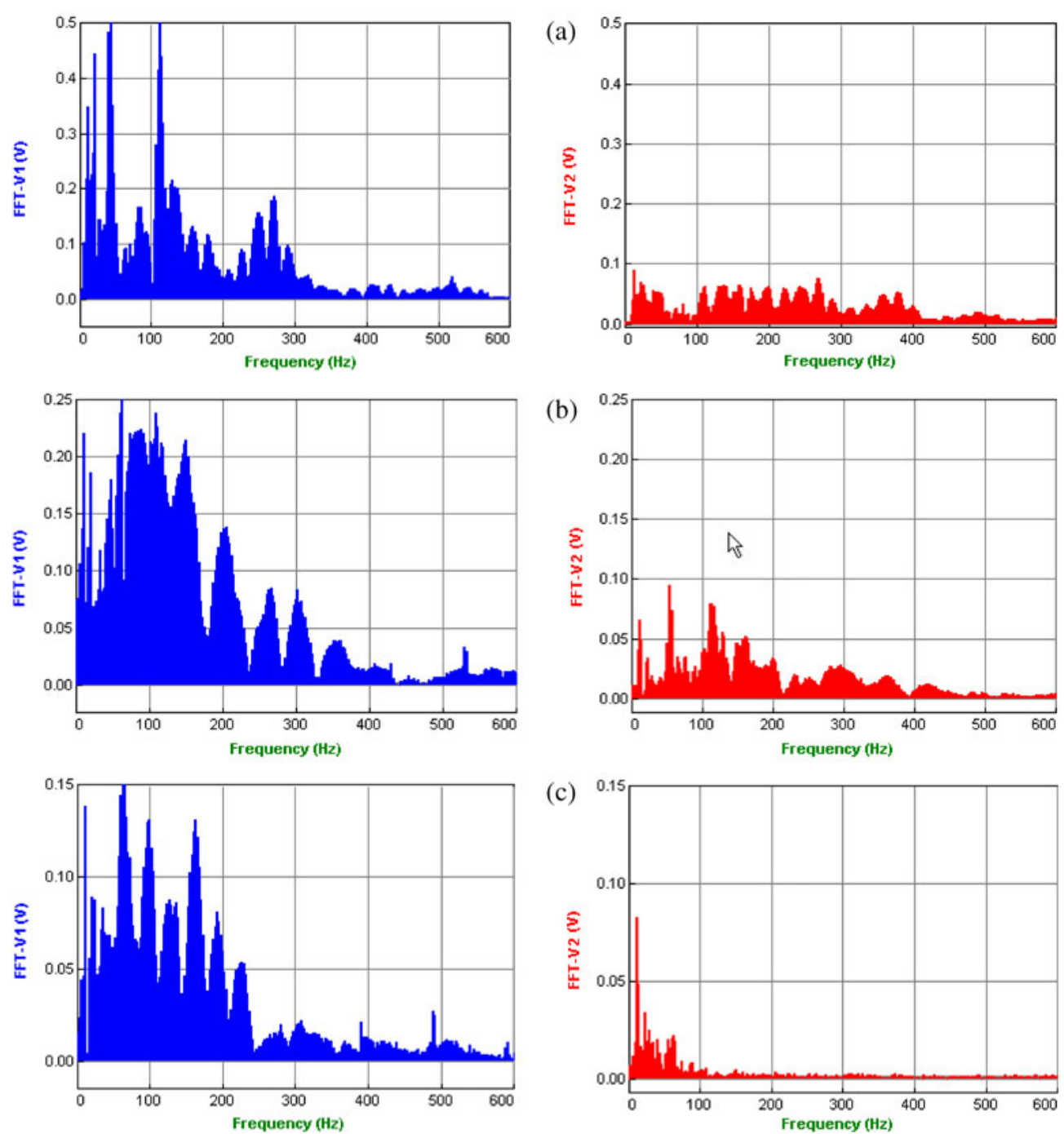

Figure 3. FFT transforms of signals detected by the two seismometers in aluminium alloy (a), wood (b) and sand (c).

can be useful to introduce students to the evidence that 'something' is propagating into the media without any macroscopic displacement of matter. Moreover, we are able to measure the velocity of the pulse propagation [8] and show that it depends on the nature of the material. As a consequence, the experiments may provide good examples in order to deduce that what is propagating is energy or a 'perturbation' in the medium: this is particularly evident in sand, where students are able to actually see the 'particles' constituting the medium.

The analysis of the results reported in table 1 can easily introduce students to the concepts related to the attenuation of perturbations propagating in media exhibiting different properties. This attenuation involves two different aspects: a geometrical aspect due to the diffusion of energy from small areas (the contact areas between the falling ball and the slabs) to the whole area of the slab and from the slab to the environment; a damping aspect due to the different 
behaviours of the molecular/atomic motion of the particles constituting the system. The first aspect can be considered almost the same in all the slabs (a consequence of their almost equal geometrical shape), but the second one is different from medium to medium since it is strongly dependent on the media characteristics.

In materials without a well-organized long-range structure, such as sand substrates or wood, one can justify only the main characteristics of the interaction at mesoscopic level. In this context, the behaviour of sand can be visualized as that of a collection of small masses interacting through inelastic collisions. When a pulse is generated, these interactions determine energy propagation together with relevant energy absorption of the shock wave. The latter effect causes not only attenuation but also deformation of the pulse. A similar qualitative behaviour can be ascribed to complex systems made up of large molecules, roughly representing the wood structure, where wave propagation is characterized by damping attributed to 'macroscopic friction'.

In materials with a long-range order such as metals, qualitative and/or semi-quantitative arguments can be used to justify the response to a shock wave. Microscopic models of a metal as a lattice of particles (atoms) and springs (representing the interactions between the particles) [17], help to visualize the different dynamic behaviour of a metal with respect to the amorphous matter.

When the lattice is perturbed by shock waves (acoustic waves), the initial vibrational energy can be distributed between different vibrational modes. This redistribution of the energy content of the pulse into other vibration modes, not active before, causes a reduction of the pulse peak, so that the wave packet is progressively deformed and attenuated. As a consequence, the attenuation of the wave pulse can be mainly attributed to the conversion of ordered motion into progressively disordered motion, involving other vibrations with different frequencies, resulting in an attenuation and deformation of the pulse with a change of its spectral distribution.

\section{Conclusion}

In conclusion, the present educational approach is aimed at introducing and developing the phenomenon of wave propagation in real systems by taking as a starting point a multidisciplinary problem involving disciplines not traditionally within current educative systems. Our results, showing that the propagation of shock waves in different materials is deeply affected by the nature of material, may help students to understand some disciplinary problems not strictly related, such as the reason why many Roman buildings are still in place today, without any major damage, even after many earthquakes have affected the zones in which they were built.

Moreover, the purpose of our approach is at least twofold. On the one hand it shows that experimental measurements, carried out using low-cost instrumentation and using friendly RTL systems, can highlight the basic background of applied physics and give answers to observed practical and real-life situations. On the other hand it can be used to introduce and/or deepen the understanding of relevant physics topics in a stimulating context.

\section{Acknowledgment}

This research was funded by MIUR (Italian Ministry of Instruction, University and Research), COFIN 2001. 


\section{Appendix. The Fourier transform}

The Fourier theorem states that a wide class of waveforms can be transformed into a superposition of a series of sine and cosine waves [10]. The Fourier transform uses the above concept to convert a function of a certain variable (say, the time) into a function of a conjugate variable (say, the frequency). In detail,

$$
\begin{aligned}
& f(t)=\frac{1}{2 \pi} \int_{-\infty}^{+\infty} F(\omega) \mathrm{e}^{-\mathrm{i} \omega t} \mathrm{~d} \omega \\
& F(\omega)=\int_{-\infty}^{+\infty} F(t) \mathrm{e}^{\mathrm{i} \omega t} \mathrm{~d} t .
\end{aligned}
$$

In these equations $\omega$ is angular frequency and $t$ is time.

The function $F(\omega)$ gives the distribution law of the harmonic components, whereas $f(t)$ describes the time dependence of the signal. Both these functions are suitable descriptions of a physical process. If the process occurs in time and is described by $f(t)$, we can find its spectral content by evaluating its transform $F(\omega)$.

Since experimental data usually consist of discrete numbers of data points rather than a continuous function, the discrete Fourier transform (DFT) (an algorithm devised for carrying out the transform with discrete data) is usually implemented in the form

$$
F_{n}=\sum_{k=0}^{N-1} f_{k} \exp \left(-2 \pi \frac{n k}{N}\right)
$$

where $F_{n}$ is the $n$th coefficient $(n=1,2, \ldots, N-1)$ of the DFT and $f_{k}$ the $k$ th sample of the temporal series consisting of $N$ samples.

The calculation of the DFT of $N$ data requires a number of operations (additions and multiplications) of the order of $N^{2}$. This algorithm has been supplanted by fast Fourier transform (FFT) algorithm, which reduces the redundancies and requires much less computer time. The order of the calculations reduces now to $N \log _{2} N$. The algorithm of the FFT is based on the reduction of the DFT in two series (each containing one half of samples) and in a process of iteration that halves each sequence. The algorithm is reported in many books of computational methods and many of them report its coding in Basic (see, for example [13] or [14]). The code translation in VBA (Visual Basic for Application) allows direct implementation on the Excel spreadsheet [18].

\section{References}

[1] Boeker E, van Grondelle R and Blankert P 2003 Environmental physics as a teaching concept Eur. J. Phys. 24 S59-68

[2] Hughes S 2001 Medical ultrasound imaging Phys. Educ. 36 468-75

[3] Mathelitsch L 2001 Outdoor physics GIREP Newsletter vol 45 pp 1-4

[4] http://www.geo.mtu.edi/UPSeis/index.html and http://www.exploratorium.edu/faultline/earthquakescience/index. html

[5] http://www.egr.msu.edu/'harichan/papers/present/svegm.pdf and references therein

[6] http://www.vernier.com

[7] http://www.pasco.com

[8] Fazio C, Sperandeo-Mineo R M and Tarantino G 2003 How did Roman buildings survive earthquakes? Phys. Educ. 38 480-4

[9] Seitz F and Turnbull D 1958 Solid State Physics: Advances in Research and Applications (New York: Academic)

[10] Papoulis A 1962 The Fourier Integral and its Applications (New York: McGraw-Hill)

[11] Cooley J W and Tkey J W 1965 An algorithm for the machine calculation of complex Fourier Ser. Math. Comput. $19297-301$

[12] Wong S M 1992 Computational Methods in Physics and Engineering (London: Prentice-Hall) 
[13] Rabiner L and Gold B 1975 Theory and Application of Digital Signal Processing (London: Prentice-Hall) p 368

[14] Smith S C 1978 The Scientist and Engineer's Guide to Digital Signal Processing (New York: McGraw-Hill) p 236

[15] Wittmann M C, Steinberg R N and Redish E F 1999 Making sense of how students make sense of mechanical waves The Physics Teacher 37 15-21

[16] Wittmann M C 2002 The object coordination class applied to wave pulses: analysing student reasoning in wave physics Int. J. Sci. Educ. 24 97-118

[17] Chabay R W and Sherwood B A 1999 Bringing atoms into first-year physics Am. J. Phys. 67 1045-50

[18] http://www.microsoft.com 